\title{
Higher Order Wave Equation with Logarithmic Source Term
}

\author{
Sh. Hajrulla1,*, L. Bezati ${ }^{1}$, F. Hoxha ${ }^{2}$ \\ ${ }^{1}$ University of Vlora, Albania, \\ 2University of Tirana, Albania
}

e-mail: shkelam.hajrulla@univlora.edu.al ${ }^{1}, \underline{\text { leonard.bezati@univlora.edu.al }}{ }^{1}$, fatmir.hoxha@fshn.edu.al $^{2}$

\begin{abstract}
In this paper we study the initial boundary value problem for logarithmic Higher Order Wave equation. Introducing the Logarithmic Sobolev inequality and using the combination of Galerkin method, we consider the theorem of existence of a global weak solution to problem for the initial boundary value problem of the logarithmic wave equation. By constructing an appropriate Lyapunov function, we obtain the decay estimates of energy for logarithmic Higher Order Wave equation. The proof of the main theorem is given.
\end{abstract}

Key words: global existence; damping; boundary source; logarithmic wave equation; initial boundary value problem; decay estimate

\section{Introduction}

In this paper, we consider the initial boundary value problem of the logarithmic wave equation

$$
\begin{array}{lc}
u_{t t}+A+u+u_{t}|u|^{2} u=u \ln |u|^{k}, & (x, t) \epsilon \Omega \times[0, \mathrm{~T}), \\
u(x, t)=0, & x \in \partial \Omega, \mathrm{t}>0 \\
u(x, 0)=u_{0}(x) \text { and } u_{t}(x, 0)=u_{1}(x), & x \in \Omega,
\end{array}
$$

where $\Omega \subset R^{n}, \mathrm{n} \geq 1$, is a bounded domain with smooth boundary $\partial \Omega, \mathrm{k} \geq 1$ and $A=(-\nabla)^{m}$,

$\mathrm{m} \geq 1$ is a natural number and $0<k<1$ is a small parameter. We deal with decay estimates of energy for (1.1) - (1.3). Hiramatsu [4] also introduced the following equation:

$$
u_{t t}-\Delta u+u+|u|^{2} u=u \ln |u|^{k},(x, t) \epsilon \Omega X(0, T), \mathrm{t}>0
$$

when studying the dynamics of Q-balls in theoretical physics. There is extensive literature on logarithmic equation used by Enzo Vitillaro [2] and Gorka P. [3], nonlinearity of waves used by Bialynicki-Birula, I., Mycielski, J. [6] , existence of solution used by Han X. S [7], the question of existence (see [8]), and asymptotic behavior of solution (see [13]) for the following initial boundary value problem

$$
\begin{array}{ll}
u_{t t}+A+u+h\left(u_{t}\right)=f(u), & x \in \Omega, \mathrm{t}>0 \\
u(x, t)=0, & x \in \partial \Omega, \mathrm{t}>0 \\
u(x, 0)=u_{0}(x) \text { and } u_{t}(x, 0)=u_{1}(x), & x \in \Omega,
\end{array}
$$


By constructing an appropriate Lyapunov function, we obtain the decay estimates of energy for logarithmic Higher Order Wave equation. So, the purpose of this paper is to obtain a decay estimate of solutions to the problem (1.1)-(1.3) in the stable set for which the solution of problem (1.1)-(1.3) decays exponentially. The key tool in the proof is an idea of Harux and Zuazua [11] and [12] and it has been recently used by Benaissa [9] and Gerbi [10]. This method is based on the construction of a suitable Lypanov function and this kind of Lypanov function is a small perturbation of the energy. By using method Gorka [3] obtained the global existence of weak solutions for all $u_{0} \in H_{0}{ }^{m}, u_{1} \in$ $L^{2}$ to the initial boundary value problem.

\section{Preliminaries and some Lemmas}

We denote by $\|.\|_{p}$ the $\mathrm{L}^{\mathrm{p}}(\Omega)$ norm, and $\| \nabla$. $\|$ the Dirichlet norm in $H_{0}{ }^{m}$. In particular, we denote $\|\|=.\|.\|_{2}$. We also use $C$ to denote a universal positive constant that may have different.

By a weak solution $u(x, t)$ of problem (1.1) $-(1.3)$ on $\Omega \times[0, T)$, we mean

$$
u \in C\left([0, T], H_{0}{ }^{m}(\Omega)\right) \cap C\left([0, T], \mathrm{L}^{2}(\Omega), u_{t t} \in C\left([0, T], \mathrm{H}^{\mathrm{m}}(\Omega)\right.\right.
$$

such that $u(x, 0)=u_{0}(x)$ in $H_{0}{ }^{m}, u_{t}(x, 0)=u_{1}(x)$ in $\mathrm{L}^{2}$ and

$$
\left(u_{t t}, w\right)+\left(A^{\frac{1}{2}} u, A^{\frac{1}{2}} w\right)+(u, w)+\left(u_{t}, w\right)+\left(|\mathrm{u}|^{2} \mathrm{u}, w\right)=\left(u \ln |u|^{k}, w\right),
$$

for any $w \in H_{0}{ }^{m}(\Omega)$, (a.e. $t \in[0, T)$ ) and the following energy inequality of the weak solutions $\boldsymbol{u}$ holds:

$$
E(t) \leq E(0), \quad \text { for all } t \in[0, T)
$$

Denote:

$$
\begin{aligned}
& E(t)=E(u)=\frac{1}{2}\left(\left\|u_{t}\right\|^{2}+\left\|A^{\frac{1}{2}} u\right\|^{2}+\|u\|^{2}-\int_{\Omega} u^{2} \ln |\mathrm{u}|^{\mathrm{k}} d x\right)+\frac{k}{4}\|u\|^{2} \\
& E(0)=\frac{1}{2}\left(\left\|u_{1}\right\|^{2}+\left\|A^{\frac{1}{2}} u_{0}\right\|^{2}+\left\|u_{0}\right\|^{2}-\int_{\Omega} u_{0}^{2} \ln \left|u_{0}\right|^{\mathrm{k}} d x\right)+\frac{k}{4}\left\|u_{0}\right\|^{2}
\end{aligned}
$$

Lemma 2.1: (Logarithmic Sobolev inequality)

Let $\boldsymbol{u}$ be any function in $H_{0}{ }^{m}(\Omega)$ and $a>0$ be any number. Then (see [1,3,5])

$$
2 \int_{\Omega}|\mathrm{u}|^{2} \ln \frac{|\mathrm{u}|}{\|u\|} d x+n(1+\ln a)\|u\|^{2} \leq \frac{a^{2}}{\pi} \int_{\Omega}\left|\frac{1}{2} u\right|^{2} d x
$$

Theorem 2.2: Suppose that $u_{0} \in H_{0}{ }^{m}(\Omega)$ and $u_{1} \in \mathrm{L}^{2}(\Omega)$, then there exists a global weak solution to problem (1.1) - (1.3).

Proof: We introduce two functional $J(u)$ and $I(u)$ :

$$
J(u)=J(u(t))=J(t)=\frac{1}{2}\left(\left\|A^{\frac{1}{2}} u\right\|^{2}+\|u\|^{2}-\int_{\Omega} u^{2} \ln |u|^{\mathrm{k}} d x\right)+\frac{1}{4}\|u\|_{4}^{4}+\frac{k}{4}\|u\|^{2}
$$




$$
I(u)=I(u(t))=I(t)=\left\|A^{\frac{1}{2}} u\right\|^{2}+\|u\|^{2}-\int_{\Omega} \mathrm{u}^{2} \ln |\mathrm{u}|^{\mathrm{k}} d x+\frac{1}{2}\|u\|_{4}^{4}
$$

We can simple see that

$$
\begin{aligned}
& J(u)=J(u(t))=\frac{1}{2} I(u)+\frac{k}{4}\|u\|^{2} \\
& E(t))=\frac{1}{2}\left\|u_{t}\right\|^{2}+J(u)
\end{aligned}
$$

We can simple see that by using this Lemma (2.1) and with the combination of Galerkin method with it, we can see the proof of the theorem. Note $w=u_{t}$ in equation 2.1 and using that $\left(u \ln u^{k}\right)=\frac{d}{d t} \frac{1}{2} u^{2} \ln |u|^{k}-\frac{k}{4} u^{2}$,

we get

$$
\frac{d}{d t} E(t)=-\left\|u_{t}\right\|^{2}
$$

Now, we define the potential depth as

$$
\boldsymbol{d}=\inf \left\{\sup J(\lambda u), \quad u \in H_{0}(\Omega) \quad 0\right\} \quad \lambda \geq 0
$$

Lemma 2.3 Let $u \in H_{0}{ }^{m}(\Omega) \backslash\{0\}$. Define the well-known Nehari manifold as follow

then

$$
N=\left\{u / u \in H_{0}^{m}(\Omega) \backslash\{0\} . I(u)=0\right\}
$$

(i) $I(\lambda u)=\lambda \frac{d}{d \lambda} J(\lambda u)$ and $\lim _{\lambda \rightarrow 0} J(\lambda u)=0, \lim _{\lambda \rightarrow+\infty} J(\lambda u)=-\infty$

(ii) There exists a unique $\lambda^{*}=\lambda(u)$ such that $\frac{d}{d \lambda} J(\lambda u) \mid \lambda=\lambda^{*}=0$, and $J(\lambda u)$ is increasing on $0<\lambda \leq \lambda^{*}$, decreasing on $\lambda^{*} \leq \lambda<+\infty$, and reaches a maximum at $\lambda=\lambda^{*}$. In other words, there exists a unique $\lambda^{*} \in(0,+\infty)$ such that $I\left(\lambda^{*} u\right)=0$ and

$$
I(\lambda u)=\lambda \frac{d}{d \lambda} J(\lambda u)>0 \text { for all } 0<\lambda \leq \lambda^{*}, I(\lambda u)<0, \lambda>\lambda^{*}
$$

where

$$
\lambda^{*}=\exp \frac{\left\|\frac{1}{2} u\right\|^{2}+\|u\|^{2}-\int_{\Omega} u^{2} \ln |\mathbf{u}|^{\mathrm{k}} d x+\frac{1}{2}\|u\|_{4}^{4}}{k\|u\|^{2}}
$$

Then, it is readily seen that the potential depth $d$ is also characterized by

$$
\boldsymbol{d}=\inf J(u) \quad u \in N
$$

Related to the problem (1.1) - (1.3) we define the following subset:

and

$$
W=\left\{u \in H_{0}{ }^{m}(\Omega) \mid J(u)<d, I(u)>0\right\} \text { is the stable set }
$$

$$
U=\left\{u \in H_{0}{ }^{m}(\Omega) \mid J(u)<d, I(u)<0\right\} \text { is the unstable set }
$$

Lemma 2.4 Let $u \in H_{0}{ }^{m}(\Omega)$ and $l=\left(\frac{2 \pi}{k}\right)^{\frac{\pi}{2}} e^{n}$.

If $0<\|u\|^{2} \leq l$, then $I(u) \geq 0$. If $I(u)=0$ and $\|u\| \neq 0,(u \in N)$ than $\|u\|^{2} \geq l$. If $I(u)<0$, than $\|u\|^{2} \geq l$. 
Lemma $2.5 d \geq \frac{k}{4}\left(\frac{2 \pi}{k}\right)^{\frac{\pi}{2}} e^{n}=\frac{k}{4} l$, where $l=\left(\frac{2 \pi}{k}\right)^{\frac{\pi}{2}} e^{n}$

Proof: $\quad$ If we take $I(u)=0$ and $\|u\|=0$, then by Lemma 2.3 we have $\|u\|^{2} \geq l$. Together with Lemma 2.4 we get

Than we have $d \geq \frac{k}{4} l$.

$$
J(u)=J(u(t))=\frac{1}{2} I(u)+\frac{k}{4}\|u\|^{2} \geq \frac{k}{4}\left(\frac{2 \pi}{k}\right)^{\frac{\pi}{2}} e^{n}
$$

Lemma 2.6 If $u_{0} \in H_{0}{ }^{m}(\Omega)$ and $u_{1} \in \mathrm{L}^{2}, 0<E(0)<d$ and $\boldsymbol{u}$ is a weak solution of problem (1.1) - (1.3) on [0,T), where $T$ is the maximal existence time of weak solution, then $u \in W$ if $I\left(u_{0}\right)<0$.

Proof: $\quad$ By the following energy inequality of the weak solutions $\boldsymbol{u}$ holds in in-equation (2.2) $E(t) \leq E(0), \quad$ for all $t \in[0, T)$,

where we have denoted:

$$
\begin{aligned}
& E(t)=E(u)=\frac{1}{2}\left(\left\|u_{t}\right\|^{2}+\left\|A^{\frac{1}{2}} u\right\|^{2}+\|u\|^{2}-\int_{\Omega} \mathrm{u}^{2} \ln |\mathrm{u}|^{\mathrm{k}} d x\right)+\frac{k}{4}\|u\|^{2} \\
& E(0)=\frac{1}{2}\left(\left\|u_{1}\right\|^{2}+\left\|A^{\frac{1}{2}} u_{0}\right\|^{2}+\left\|u_{0}\right\|^{2}-\int_{\Omega} u_{0}^{2} \ln \left|u_{0}\right|^{\mathrm{k}} d x\right)+\frac{k}{4}\left\|u_{0}\right\|^{2}
\end{aligned}
$$

we have

$$
E(t))=\frac{1}{2}\left\|u_{t}\right\|^{2}+J(u) \leq \frac{1}{2}\left\|u_{1}\right\|^{2}+J\left(u_{0}\right)<d
$$

\section{Decay estimates of the solution}

In this section we prove that the solution will grow as an exponential function in the $H_{0}{ }^{\mathrm{m}}(\Omega)$ norm as time goes to infinity for small positive initial energy or $E(0)<0$.

Theorem 3.1 Let $\mathrm{u}_{0} \in W, \mathrm{u}_{1} \in \mathrm{L}^{2}(\Omega)$ and $0<E(0)<\frac{k}{4} 4 l<d$, then the solution of (1.1)(1.3) grows as an exponential function in the $H_{0}{ }^{\mathrm{m}}(\Omega)$ norm.

Theorem 3.2 Let $\mathrm{u}_{0} \in W(\Omega), \mathrm{u}_{1} \in \mathrm{L}^{2}(\Omega)$. Let $0<E(0)<\frac{\mathrm{ck}}{4}<\mathrm{d}$ where $\boldsymbol{c}$ is a constant such that $0<k^{\frac{4}{\mathrm{n}}} C^{\frac{4}{\mathrm{n}}} \frac{2 \pi}{\mathrm{k}} e^{2}<1$. Then, there exist two positive constants $K$ and $\gamma$ independent of $t$, such that:

$$
0<E(\mathrm{t})<\mathrm{K} e^{-\gamma t}<\mathrm{d}, \quad \mathrm{t} \geq 0
$$

Proof: Let we note $H(t)=-E(t)$. Let $u(t, x)$ be a weak solution of problem (1.1) - (1.3). Since $\mathrm{u}_{0} \in, \mathrm{u}_{1} \in \mathrm{L}^{2}(\Omega)$ as we have explained in section 2 , we have

$$
\mathrm{u} \in W \text { for all } \mathrm{t} \in(0,+\infty) \text { and } 0<E(\mathrm{t})<\mathrm{d} \text { and } I(u)>0
$$

Now, we construct the Lyapunov functional by performing a suitable modification of the energy

$$
L(t)=E(t)+\varepsilon\left(u, u_{t}\right) \quad(\text { where } \varepsilon>0) .
$$


By the definition of $E(t)$ and since $\left|\left(\mathrm{u}, u_{t}\right)\right| \leq \frac{1}{2}\left(\|u\|^{2}+\left\|u_{t}\right\|^{2}\right)$, than we know that $E(t)$ and $L(t)$ are equivalent in the sense that there exist two positive constants $\beta_{1}$ and $\beta_{2}$ depending on $\varepsilon$ such that

$$
\beta_{1} E(t) \leq \mathrm{L}(\mathrm{t}) \leq \beta_{2} E(t)
$$

By taking the time derivative of the function $L(t)$ and performing the integration by parts than we get

$$
L^{\prime}(t)=(\varepsilon-1)\left\|u_{t}\right\|^{2}+\varepsilon\left\|A^{\frac{1}{2}} u\right\|^{2}-\varepsilon\|u\|^{2}-\varepsilon\|u\|_{4}^{4}-\varepsilon\left(\mathrm{u}, u_{t}\right)+\varepsilon \int_{\Omega} \mathrm{u}^{2} \ln |\mathrm{u}|^{\mathrm{k}} d x
$$

$L^{\prime}(t)=\left(1+\varepsilon+\varepsilon \frac{\mathrm{M}}{2}\right)\left\|u_{t}\right\|^{2}+\varepsilon \frac{\mathrm{M}-2}{2}+\left\|A^{\frac{1}{2}} u\right\|^{2}+\varepsilon \frac{\mathrm{M}-2}{2}+\|u\|^{2}-\varepsilon \frac{\mathrm{M}-2}{2} \int_{\Omega} \mathrm{u}^{2} \ln |\mathrm{u}|^{\mathrm{k}} d x+\varepsilon M H(t)+$ $\frac{k}{4}\|u\|^{2}$

and since $E(t) \leq E(0) \leq 0$ by the definition of $E(t)$, we know that $\int_{\Omega} \mathrm{u}^{2} \ln |\mathrm{u}|^{\mathrm{k}} d x>0$

Than

$$
\begin{gathered}
L^{\prime}(t) \geq \theta\left[\left\|u_{t}\right\|^{2}+\left\|A^{\frac{1}{2}} u\right\|^{2}+\|u\|^{2}+H(t)\right], \\
H(t)=-E(t) \leq \int_{\Omega} \mathrm{u}^{2} \ln |\mathrm{u}|^{\mathrm{k}} d x
\end{gathered}
$$

Now, using known Young inequality we obtain

$$
\left|\left(\mathrm{u}, u_{t}\right)\right| \leq \frac{1}{4 \delta}\left(\left\|u_{t}\right\|^{2}+\delta\|u\|^{2}\right) \text { for } \delta>0
$$

Using the definition and inserting (3.3) and (3.4), we have

$$
\begin{aligned}
& L^{\prime}(t) \leq-M \varepsilon \mathrm{E}(\mathrm{t})+\left(\frac{\mathrm{M} \varepsilon}{2}+\varepsilon+\frac{\varepsilon}{4 \delta}-1\right)\left\|u_{t}\right\|^{2}+M \varepsilon \mathrm{E}(\mathrm{t})+\varepsilon\left(\frac{\mathrm{M}}{2}-1\right)\left\|A^{\frac{1}{2}}\right\|^{2}+ \\
& \varepsilon\left(\frac{\mathrm{M}}{2}+\delta-1\right)\|u\|^{2}+\frac{\mathrm{Mk}}{4}\|u\|^{2}+\varepsilon\left(\frac{\mathrm{M}}{4}-1\right)\|u\|_{4}^{4}+\varepsilon\left(1-\frac{\mathrm{M}}{2}\right) \int_{\Omega} \mathrm{u}^{2} \ln |\mathrm{u}|^{\mathrm{k}} d x
\end{aligned}
$$

Recall now the logarithmic Sobolev inequality, for $0 \leq M \leq 1$

$$
\begin{aligned}
L^{\prime}(t) \leq- & M \varepsilon E(t)+\left(\frac{\mathrm{M} \varepsilon}{2}+\varepsilon+\frac{\varepsilon}{4 \delta}-1\right)\left\|u_{t}\right\|^{2}+\varepsilon\left(\frac{\mathrm{M}}{2}-1\right)\left\|A^{\frac{1}{2}}\right\|^{2}+\varepsilon\left(1-\frac{\mathrm{M}}{2}\right) \frac{\mathrm{k} \alpha^{2}}{2 \pi}\left\|A^{A^{\frac{1}{2}}}\right\|^{2}+ \\
& \varepsilon\left(\frac{\mathrm{M}}{2}+\delta-1\right)\|u\|^{2}+\left(k \varepsilon\left(\frac{\mathrm{M}}{4}-1\right) \ln \|u\|^{2}\right)\|u\|^{2}-\varepsilon n k\left(1-\frac{\mathrm{M}}{2}\right) \frac{1+\ln \mathrm{a}}{2}\|u\|^{2} \\
= & L^{\prime}(t) \leq-M \varepsilon \mathrm{E}(\mathrm{t})+\left(\frac{\mathrm{M} \varepsilon}{2}+\varepsilon+\frac{\varepsilon}{4 \delta}-1\right)\left\|u_{t}\right\|^{2}-\varepsilon\left(1-\frac{\mathrm{M}}{2}\right)\left(1-\frac{\mathrm{k} \alpha^{2}}{2 \pi}\right)\left\|A^{\frac{1}{2}}\right\|^{2}+ \\
& \left(\frac{\mathrm{M}}{2}+\delta-1+\mathrm{k}\left(1-\frac{\mathrm{M}}{2}\right) \ln \|u\|^{2}-n k\left(1-\frac{\mathrm{M}}{2}\right) \frac{1+\ln \mathrm{a}}{2}\right)\|u\|^{2}
\end{aligned}
$$

Noting $1-M / 2>0$ since $0<M<1$, and $\ln \|u\|^{2}<\ln \|u\|^{2}(4 J(u))$, we get 


$$
\begin{aligned}
& L^{\prime}(t) \leq-M \varepsilon \mathrm{E}(\mathrm{t})+\left(\frac{\mathrm{M} \varepsilon}{2}+\varepsilon+\frac{\varepsilon}{4 \delta}-1\right)\left\|u_{t}\right\|^{2}-\varepsilon\left(1-\frac{\mathrm{M}}{2}\right)\left(1-\frac{\mathrm{k} \alpha^{2}}{2 \pi}\right)\left\|A^{\frac{1}{2}}\right\|^{2} \\
&+\varepsilon\left(\frac{\mathrm{M}}{2}+\delta-1+\mathrm{k}\left(1-\frac{\mathrm{M}}{2}\right) \ln (4 J(u))-n k\left(1-\frac{\mathrm{M}}{2}\right) \frac{1+\ln \mathrm{a}}{2}\right)\|u\|^{2} \\
&=-M \varepsilon \mathrm{E}(\mathrm{t})+\left(\frac{\mathrm{M} \varepsilon}{2}+\varepsilon+\frac{\varepsilon}{4 \delta}-1\right)\left\|u_{t}\right\|^{2}-\varepsilon\left(1-\frac{\mathrm{M}}{2}\right)\left(1-\frac{\mathrm{k} \alpha^{2}}{2 \pi}\right)\left\|A^{\frac{1}{2}}\right\|^{2}+ \\
& \varepsilon\left(\frac{\mathrm{M}}{2}+\delta-1+\mathrm{k}\left(1-\frac{\mathrm{M}}{2}\right)\left(\ln (4 J(u))-n \frac{1+\ln \mathrm{a}}{2}\right)\right)\|u\|^{2}
\end{aligned}
$$

Since $0 \leq M \leq 1, J(u)<0<\frac{\mathrm{k} \alpha \mathrm{l}}{4}$ by satisfying $0<k^{\frac{4}{\mathrm{n}}} c^{\frac{4}{\mathrm{n}}} \frac{2 \pi}{\mathrm{k}} e^{2}<a^{2}<\frac{2 \pi}{\mathrm{k}}$

where $a$ can be assured by the assumption about $\alpha$ in Theorem 3.1, and taking $\delta>0$ small sufficiently such that $-1-\frac{1-a^{2}}{2 \pi}>0, \frac{\mathrm{M}}{2}+\delta-1+\mathrm{k}\left(1-\frac{\mathrm{M}}{2}\right)\left(\ln \left(4 J(u)-\frac{\mathrm{n}(1+\ln a)}{2}\right)<0\right.$

$$
L^{\prime}(t) \leq-M \varepsilon \mathrm{E}(\mathrm{t})+\left(\frac{\mathrm{M} \varepsilon}{2}+\varepsilon+\frac{\varepsilon}{4 \delta}-1\right)\left\|u_{t}\right\|^{2}
$$

Let we take $\varepsilon>0$ small sufficiently such that $\frac{\mathrm{M} \varepsilon}{2}+\varepsilon+\frac{\varepsilon}{4 \delta}-1<0$

So, the inequality (3.9) become

$L^{\prime}(t) \leq-M \varepsilon E(t)$

Using (3.2), we have

$$
L^{\prime}(t) \leq-M \beta_{2} L(\mathrm{t})
$$

Setting $K=M \varepsilon E(t)$ and integrating the inequality (3.10) between 0 and $t$ gives as follow

$$
L^{\prime}(t) \leq C e^{K t}
$$

This complete the proof of the Theorem 3.2.

\section{Conclusion}

The idea of using damping to positively influence and properties of solutions was introduced more than 20 years ago in the context of control theory for boundary or point controls, which by themselves, without any damping. In contrast to systems on $R^{n}$, here where $\Omega \subset R^{n}, \mathrm{n} \geq 1$, is a bounded domain with smooth boundary $\partial \Omega, \mathrm{k} \geq 1$ and $A=(-\nabla)^{m},(m \geq 1)$. By the construction of a apropriate Lypanov function as a small perturbation of the energy and using method Gorka we obtained the global existence of weak solutions for the higher order water wave equations with logarithmic term to the initial boundary value problem. The equations are characterized by the interplay of two competing nonlinearities:

(i) nonlinear sources, especially of higher order above the super-critical exponent; instead such sources build up the energy and drive the solution to a blow up in finite time.

(ii) a nonlinear damping on the interior/boundary whose role becomes two-fold: it is intended not only to stabilize the system, as in the classical control theory for dissipative dynamics, but also to prevent the finite time blow-up of solution and guarantee its existence and uniqueness. 
The interaction of source and damping can take place in the interior of the physical domain and/or in the boundary conditions.

Acknowledgements: This article is supported by Prof. E. Pişkin of Department of Mathematics in Dicle University, Turkey and the Natural Science Faculty of University of Tirana, Albania.

\section{References}

[1] Enzo Vitillaro. Global existence for the wave equation with nonlinear boundary damping and source terms. J. Differential Equations , 186(1):259-298, 2002.

[2] Enzo Vitillaro. A potential well theory for the wave equation with nonlinear source and boundary damping terms. Glasg. Math. J., 44(3):375-395, 2002.

[3] Gorka P., Logarithmic Klein-Gordon equation. Acta Phys. Polon. B, 40(1) (2009), 59-66.

[4] Hiramatsu, T., Kawasaki, M., Takahashi, F.: Numerical study of Q-ball formation in gravity mediation.

[5] Gross L., Logarithmic Sobolev inequalities. Amer. J. Math., 97(4) (1975), 1061-1083.

[6] Bialynicki-Birula, I., Mycielski, J.: Nonlinear wave mechanics. Ann. Phys. 100(1-2), 62-93 (1976)

[7] Han X. S., Global existence of weak solutions for a logarithmic wave equation arising from Q-ball dynamics. Bull. Korean Math. Soc., 50(1) (2013), 275-283.

[8] Chen H., Luo P. and Liu G.W., Global solution and blow-up of a semilinear heat equation. with logarithmic nonlinearity. J. Math. Anal. Appl., 422 (1) (2015), 84-98.

[9] Benaissa A., Messaoudi S., Exponential decay of solutions of a non-linearly damped wave equation. Nonlinear Differential Equations Appl., 12(4) (2005), 391-399

[10] Gerbi S., Said-Houari B., Exponential decay for solutions to semilinear damped wave equation. Discrete and Continuous Dynamical Systems -Series S, 5(3) (2012), 559-566.

[11] Haraux A., Zuazua E., Decay estimates for some semilinear damped hyperbolic problems Arch. Ration. Mech. Anal., 150 (1988), 191-206.

[12] Zuazua E., Exponential decay for the semilinear wave equation with locally distributed damping. Comm. Partial. Diff. Eq., 15(2) (1990), 205-235.

[13] Liu, W.J.: Global existence, asymptotic behaviour and blow-up of solutions for coupled KleinGordon equations with damping terms. Nonlinear Anal. 73, 244-255 (2010) 